\title{
The gait is less stable in children with cerebral palsy in normal and dual-task gait
}

Harri Piitulainen $^{1,2,3}$, Juha-Pekka Kulmala ${ }^{1}$, Helena Mäenpää ${ }^{1}$, Timo Rantalainen ${ }^{3}$

${ }^{1}$ Motion Analysis Laboratory, Helsinki University Hospital (HUH) Children and Adolescents, Helsinki, Finland

${ }^{2}$ Department of Neuroscience and Biomedical Engineering, Aalto University School of Science, Espoo, Finland

${ }^{3}$ Faculty of Sport and Health Sciences, University of Jyväskylä, Jyväskylä, Finland

\section{Introduction}

Children with cerebral palsy (CP) have various gait impairments, but there is limited evidence about their gait stability. The gait has shown to be less stable in hemiplegic (HP) children compared to typically developed (TD) controls ${ }^{1}$. Some gait kinematics are also altered more in CP than TD by concurrent motor $^{2}$ and cognitive ${ }^{3}$ tasks.

\section{Research Question}

We examined whether gait complexity (i.e. stability) differs between HP, diplegic (DP) and TD children, and whether cognitive and motor dual tasks alter it.

\section{Methods}

We recorded kinematic data using inertial measurement unit (IMU; on mid-back at L3-L5 level) from 18 children with HP (13.5 \pm 2.4 years) and 12 with DP (13.0 \pm 2.1 years), and 31 TD ( $13.5 \pm 2.2$ years) during unconstrained gait, and motor (carrying a tray) and cognitive (word naming) task constrained gait at preferred speed ( 400 steps/task).

Step duration (from heel-strike to contralateral heel-strike), its standard deviation (SD) and refined compound multiscale entropy (RCME; 20 temporal-coarseness scales) indexes separately for vertical and resultant horizontal accelerations were computed. RCME were further summarized with principalcomponent analysis.

\section{Results}

Between groups. Step duration was similar in all groups $(p>0.586)$, but its variation was higher than TD in HP for all tasks $(p<0.001-0.01)$, and DP for normal $(p=0.013)$ and motor $(p=0.007)$ tasks.

The gait complexity was greater for HP $(p<0.01-0.05)$ and DP $(p<0.001-0.01)$ in all tasks and directions than TD, apart from the vertical direction during normal gait in HP $(p=0.059)$. The difference was observed at several coarseness scales especially in vertical direction and cognitive task. Furthermore, DP showed more complex gait than HP in some coarseness scales in vertical direction $(p<0.05-0.001)$, predominantly during cognitive task. No further statistically significant differences were observed between the patient groups. 
Between tasks. Step duration was longer for the dual-tasks only in TD group $(p<0.001)$, but its variation was higher for all groups $(p<0.01-0.001)$. The gait complexity did not differ significantly between the tasks within the groups.

Dual-task cost. The gait complexity increased more from the normal to dual-tasks in CP groups compared to TD group $(p<0.05)$. In DP, this was significant for all tasks and directions $(p<0.01-0.05)$, except for the horizontal direction of the motor task $(p=0.745)$. In HP, only the horizontal direction of the cognitive task showed significantly higher increase from the normal gait compared to TD ( $p=0.049)$. Similar effect was not observed for step duration ( $p>0.361$ ) or its variation $(p>0.385)$.

\section{Discussion}

The gait in children with CP is less stable compared to TD controls. The dual-task cost was stronger for children with $\mathrm{CP}$ indicating that attentional and motor load hinders more the gait stability in children with CP compared to TD controls. This means that the gait is less 'automated', and thus more attention and cortical resources are needed to compensate the impaired gait in children with CP.

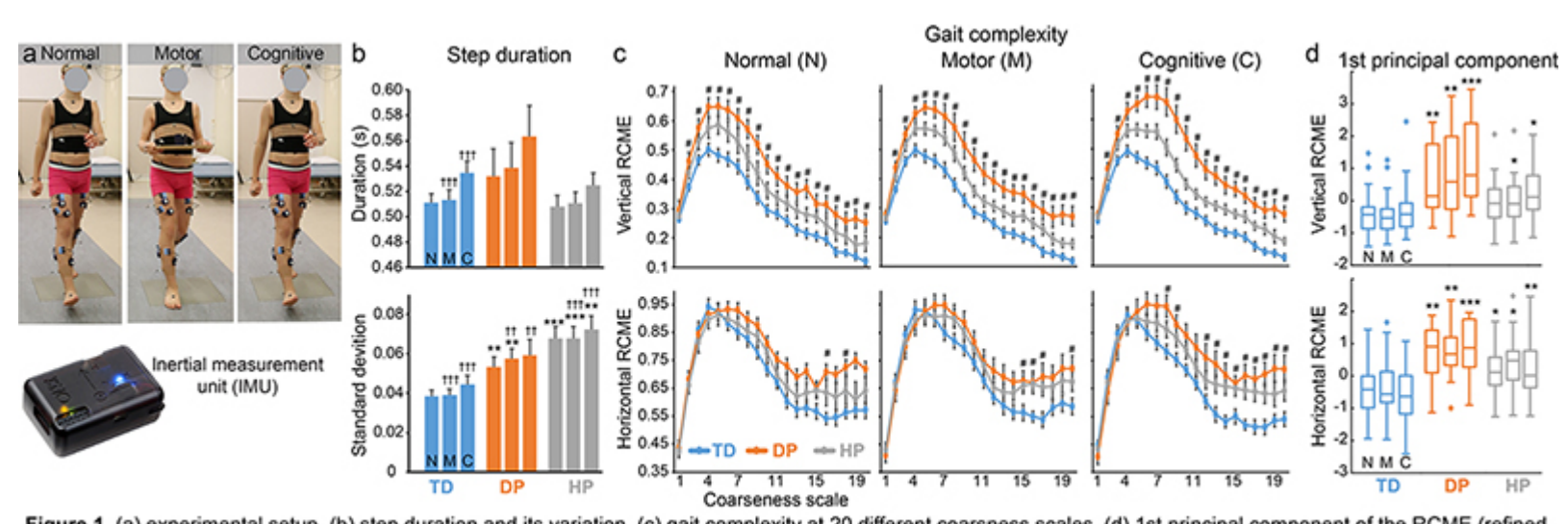

Figure 1. (a) experimental setup. (b) step duration and its variation. (c) gait complexity at 20 different coarsness scales. (d) 1 st principal component of the RCME (refined compound multiscale entropy). $* *, \cdots=$ diffrent from TD group at $p<0.05, p<0.01, p<0.001 . t \dagger, t \dagger \dagger=$ different from normal gait at $p<0.01, p<0.001 . \#=$ difference between the three groups at $p<0.05$. Non-parametric tests with Holm-Bonferoni correction were used.

\section{Keywords}

\section{CEREBRAL PALSY; DUAL-TASK; GAIT VARIABILITY; KINEMATICS; STABILITY; WALKING}

\section{Disclosure statement}

This abstract is financially supported by grants from the Academy of Finland (grants \#296240, \#326988, \#307250 and \#327288) Jane and Aatos Erkko Foundation to Harri Piitulainen.

\section{References}

1. Bruijn et al. 2013. Gait stability in children with Cerebral Palsy. Res Dev Disabil. 34:1689-99

2. Hung \& Meredith 2014. Influence of dual task constraints on gait performance and bimanual coordination during walking in children with unilateral cerebral palsy. Res Dev Disabil. 35:755-60

3. Katz-Leurer et al. 2014. Effect of concurrent cognitive tasks on temporo-spatial parameters of gait among children with cerebral palsy and typically developed controls. Dev Neurorehabil. 17:363-7 